\title{
ORAL CANDIDIASIS AS A LOCAL ADVERSE EFFECT OF INHALED CORTICOSTEROIDS: WHAT THE DENTAL PRACTITIONER SHOULD KNOW
}

\author{
Birsay GUMRU1*, Melda Pelin AKKITAP1 \\ ${ }_{1}^{1}$ Marmara University, Faculty of Dentistry, Department of Oral and Maxillofacial Radiology, 34854, Istanbul, Turkey
}

\begin{abstract}
Inhaled corticosteroids are increasingly recommended at all stages of asthma in all age groups as a first-line treatment for controlling symptoms and minimizing oral corticosteroid dependence in chronic asthma owing to their anti-inflammatory and immunosuppressive effects. Despite the fact that they are highly effective, their use can be accompanied by systemic and local adverse effects. Systemic adverse effects are infrequent, but oral candidiasis, the most common oral fungal infection, is a frequently observed local adverse effect of inhaled corticosteroid use. This adverse effect may lead to discomfort and cause reduced patient compliance. In this review, clinical findings of oral candidiasis, potential pathogenity mechanisms following such therapy along with the specific prophylactic measures that should be undertaken to minimize this adverse effect are discussed extensively. Understanding the factors leading to increased risk can give the opportunity of focusing on the patients who need timely intervention.
\end{abstract}

Keywords: Corticosteroid, Inhaler, Local adverse effects, Oral candidiasis

*Corresponding author: Marmara University, Faculty of Dentistry, Department of Oral and Maxillofacial Radiology, 34854, Istanbul, Turkey

E mail: bgumru@marmara.edu.tr (B. GUMRU)

$\begin{array}{lll}\text { Birsay GUMRU } & \text { (iD) https://orcid.org/0000-0002-7734-4755 Received: May 27, 2021 }\end{array}$

Melda Pelin AKKITAP (iD) https://orcid.org/0000-0001-7744-6615 Accepted: June 05, 2021

Published: January 01, 2022

Cite as: Gumru B, Akkitap MP. 2022. Oral candidiasis as a local adverse effect of inhaled corticosteroids: what the dental practitioner should know. BSJ Health Sci, 5(1): 107-115.

\section{Introduction}

Corticosteroids are the most effective and decent agents among anti-inflammatory drugs and play a crucial role in the effective management of asthma since they are able to interfere with several pathways implicated in the process of inflammation (Derendorf et al., 2006; Rachelefsky et al., 2007; Ullah et al., 2016; Ming et al., 2019). This fact causes use of inhaled corticosteroids (ICs) at high doses and for long time periods to a great extent (Maxwell, 1990; Hanania et al., 1995; Lipworth, 1995; Roland et al., 2004; Irwin and Richardson, 2006). Corticosteroids bind to regulators that are responsible for controlling the transcription of various proinflammatory gene products in the cell nucleus and are therefore efficient in asthma control via suppressing the inflammation carried out by these several mediators. Their action mechanism is considered to start with diffusion across the cell membrane followed by binding to glucocorticoid receptors located in the cytoplasm of the target cells (Barnes and Adcock, 2003). This binding contributes to an activated complex of glucocorticoidreceptor-corticosteroid which afterwards translocates across the nuclear membrane and binds to specific DNA sequences (Yudt and Cidlowski, 2002). Consequently, transcription of genes and synthesis of proteins are changed. Reduction in airway inflammation and hyperresponsiveness is achieved through corticosteroid usage, and a clinically considerable refinement in symptoms of asthma is ensured by change in the production of inflammatory cell-associated mediators namely macrophages, eosinophils, lymphocytes, mast cells, and dendritic cells in the airways (Maxwell, 1990; Barnes and Adcock, 2003; Fukushima et al., 2003; Roland et al., 2004; Derendorf et al., 2006; Rachelefsky et al., 2007; Dekhuijzen et al., 2016; Hossny et al., 2016; Erdoğan et al., 2019).

Inhalation is the favoured administration route of corticosteroids in the management of asthma and provides delivery of the agent to the lungs directly. The local action in the lungs minimizes or eliminates the systemic adverse effects related to oral or parenteral route (Spector et al., 1982; Hanania et al., 1995; Derendorf et al., 2006). Many steroid inhaler preparations, including beclomethasone dipropionate, fluticasone propionate, budesonide, triamcinolone acetonide, ciclesonide, betamethasone valerate, and mometasone furoate, are commercially available (Ellepola and Samaranayake, 2001; Baptist and Reddy, 2009). Low-dose ICs are prescribed as first-line treatment in management of mild persistent asthma, whereas medium-dose ICs or combination therapy using long-acting $\beta 2$-agonists is the favoured management for moderate asthma. In addition, combination therapy using high-dose ICs is suggested only in severe persistent asthma cases which are poorly controlled by combination therapy using medium-dose ICs (Dahl, 
2006; Derendorf et al., 2006; Ullah et al., 2016).

In this review; mechanisms of pathogenicity, clinical presentation, treatment, and preventive strategies of oral candidiasis, which is one of the potential local adverse effects of ICs, are discussed.

\section{Potential Local Adverse Effects of Inhaled Corticosteroids and Related Factors}

Long-term use of ICs at high doses raises concerns about local and systemic adverse effects (Table 1). Potential adverse effects associated with IC therapy may be systemic as a result of entrance of the drug into the circulation across the lungs and the gastrointestinal tract, or local due to the deposition of the drug which is actively inhaled during administration in the oral cavity and oropharynx (Toogood et al., 1980; Maxwell, 1990; Selroos et al., 1994; Hanania et al., 1995; Roland et al., 2004; Buhl, 2006; Dahl, 2006; Derendorf et al., 2006; Irwin and Richardson, 2006; Rachelefsky et al., 2007; Godara et al., 2011; van Boven et al., 2013; Hejazi et al., 2016; Patil et al., 2016; Ullah et al., 2016; Erdoğan et al., 2019; Ming et al., 2019).

Table 1. Possible local and systemic adverse effects of inhaled corticosteroids (Data taken and modified from Toogood et al., 1980; Maxwell, 1990; Selroos et al., 1994; Hanania et al., 1995; Roland et al., 2004; Buhl, 2006; Dahl, 2006; Derendorf et al., 2006; Irwin and Richardson, 2006; Rachelefsky et al., 2007; Godara et al., 2011; van Boven et al., 2013; Hejazi et al., 2016; Ullah et al., 2016; Erdoğan et al., 2019; Ming et al., 2019).

\begin{tabular}{ll}
\hline Local adverse effects & \multicolumn{1}{c}{ Systemic adverse effects } \\
\hline Bronchospasm & Adrenal insufficiency and crisis (suppressed basal cortisol, ACTH, and CRH) \\
Dysphonia & Posterior subcapsular cataracts \\
Oral candidiasis & Glaucoma \\
Pharyngitis & Decreased length of lower leg in children \\
Reflex cough & Suppressed velocity of growth in children \\
Sore throat & Reduction in bone density \\
Hoarseness of voice & Osteoporosis \\
Xerostomia & Bone fractures \\
Gingivitis and periodontitis & Thinning and bruising of skin \\
Altered taste perception & \\
Halitosis & \\
Dental caries/erosion & \\
Tongue & \\
burning/abrasion/hypertrophy & \\
Perioral dermatitis & \\
ACTH= adrenocorticotropic hormone, CRH= corticotropin-releasing hormone
\end{tabular}

When inhaled, a substantial part of the dose accumulates in the mouth and pharynx, where it has a high potential for local adverse effects. This deposited dose may be swallowed and afterwards absorbed through the gastrointestinal tract, if not rinsed off the mouth. Following its absorption from the gastrointestinal tract, the drug escapes from inactivation through the first-pass metabolism of the liver, enters the systemic circulation without any change, and potential extra-pulmonary adverse effects are encountered. Although the part of the IC dose reaching the lungs shows the expected pharmacological effect, most of the inhaled dose reaching the airways may enter the general circulation via the pulmonary vasculature, thereby causing systemic adverse effects (Derendorf, 1997; Pedersen and O’Byrne, 1997).

It is considered that the local adverse effects of ICs cause rare and minor problems, but have clinical significance, in comparison to systemic adverse effects. The local adverse effects of the ICs do not result in significant morbidity but they may compromise compliance with the therapy, causing uncontrolled asthma and a decrease in patient's life quality (Hanania et al., 1995; Roland et al., 2004; van Boven et al., 2013; Hejazi et al., 2016; Ullah et al., 2016).

The type and dose of IC used, the frequency of intake, the pharmacokinetic and pharmacodynamic properties, the type of inhaler device, and the compliance of the patient with the instructions for administration are regarded as the factors that determine the frequency of commonly observed local adverse effects of ICs (Toogood et al., 1980; Hanania et al., 1995; Derendorf, 1997; Kennedy et al., 2000; Fukushima et al., 2003; Komiyama et al., 2004; Roland et al., 2004; Buhl et al., 2006; Derendorf et al., 2006; Rachelefsky et al., 2007; van Boven et al., 2013; Dekhuijzen et al., 2016; Hejazi et al., 2016; Erdoğan et al., 2019; Ming et al., 2019).

2.1. Daily Dose, Frequency of Intake, and Treatment Duration

Increased daily dose, frequency of intake, and treatment duration are known to be positively associated with the emergence of potential adverse effects of ICs (Maxwell, 
1990; Kennedy et al., 2000; Fukushima et al., 2003; Rachelefsky et al., 2007; Thomas et al., 2010; van Boven et al., 2013; Sharma et al., 2018; Ming et al., 2019).

A study on the frequency of intake reported that the incidence of these adverse effects decreased with twice a day regimen compared to four times a day (Toogood and Jennings, 1980). Another study regarding the duration of treatment revealed that, in the first 3 months of IC use the relative risk was highest and remained increased up to 1 year after the IC onset (van Boven et al., 2013). The IC dose should be adjusted periodically to maintain asthma control with the lowest frequent dosing (Hanania et al., 1995; Rachelefsky et al., 2007).

\subsection{Pharmacokinetic and Pharmacodynamic} Properties

Various pharmacokinetic and pharmacodynamic parameters such as small particle size, high glucocorticoid-receptor-binding, high protein-binding, on-site activation in the lung, rapid systemic clearance, negligible oral bioavailability, long pulmonary residence time, high lipophilicity, high lipid conjugation, and low oropharyngeal exposure may enhance the efficacy and safety profile of an IC in asthma treatment. The antiinflammatory effects of ICs may be increased or prolonged by these aforementioned properties (Hanania et al., 1995; Derendorf, 1997; Dahl, 2006; Derendorf et al., 2006).

\subsubsection{Particle size}

Particle size is a significant factor that determines the rate at which ICs are deposited in the lower airways or the oropharyngeal cavity. The internal perimeter of the smallest airway is $\leq 2 \mu \mathrm{m}$. Particles of $<5 \mu \mathrm{m}$ are mostly deposited in bronchi and bronchioles, particles of 5-10 $\mu \mathrm{m}$ in the trachea and large bronchi, whereas particles of $>5 \mu \mathrm{m}$ mainly in the oropharyngeal cavity which may cause oral candidiasis and hoarseness as local adverse effects (Toogood et al., 1980; Maxwell, 1990; Hanania et al., 1995; Roland et al., 2004; Derendorf et al., 2006; Rachelefsky et al., 2007; Baptist and Reddy, 2009; Hossny et al., 2016). Therapeutic aerosols are generally manufactured to produce particles having a diameter of 1 to $5 \mu \mathrm{m}$ (Roland et al., 2004; Baptist and Reddy, 2009; Hossny et al., 2016).

\subsubsection{Receptor-binding affinity}

Due to the fact that some commonly expressed receptors mediate both the positive effects in the lung and the local and systemic adverse effects, receptor-binding affinity has a determining importance in the clinical safety profile of ICs. It means that high receptor-binding affinity not only increases the therapeutic ratio and the clinical effectiveness of the ICs, but also enhances their antiinflammatory activity in the lung and undesirable adverse effects by initiation of increased gene transcription, decreased gene transactivation or transexpression in all tissues (Derendorf et al., 2006).

\subsubsection{Protein-binding}

The protein-binding degree, the extent of which ranges between $71-99 \%$ for currently available ICs, is associated with controlling amount of free systemic IC and controls systemic adverse effects because only the free drug exhibits pharmacologic activity. Accordingly, as the protein-binding capacity of an IC increases, the potential for systemic adverse effects decreases (Derendorf et al., 2006; Irwin and Richardson, 2006; Baptist and Reddy, 2009).

\subsubsection{On-site activation in the lung}

On-site activation, which limits the presence of the active drug outside the target tissue, is a critical parameter that reduces the potential local adverse reactions. Many ICs such as fluticasone propionate and budesonide are inhaled in their pharmacologically active forms. Other ICs which include ciclesonide and beclomethasone 17monopropionate are activated by esterases in the lungs after inhalation as inactive compounds (Derendorf et al., 2006; Hejazi et al., 2016; Erdoğan et al., 2019).

\subsubsection{Systemic clearance}

Following inhalation, the swallowed ICs are absorbed in the gut and the circulating drug amount is reduced considerably by the hepatic first-pass metabolism (Irwin and Richardson, 2006). In addition, the removal rate of a drug from the body is important, because if the metabolism is faster the concentration of the drug decreases and the systemic adverse effect risk is reduced (Baptist and Reddy, 2009). The safety profile of an IC with a long half-life at low concentrations is likely to be better compared to an IC with a short half-life at high concentrations. Furthermore, following the systemic absorption, ICs are immediately cleared by many organs, especially the liver (Derendorf, 1997; Derendorf et al., 2006).

\subsubsection{Oral bioavailability}

Oral bioavailability is determined by the amount of the dose reaching the systemic circulation and displays both the absorbed fraction and the amount that escapes the first-pass metabolism, while pulmonary bioavailability is the part that is transmitted to the lungs and finally absorbed. As well as being essential for efficacy, high pulmonary bioavailability increases both the systemic absorption and the potential for undesirable adverse effects. In addition, if the oral bioavailability of an IC is high, the systemic absorption and potential for adverse effects are increased which is an undesirable outcome in terms of safety (Derendorf, 1997; Dahl, 2006; Derendorf et al., 2006; Baptist and Reddy, 2009).

\subsubsection{Pulmonary residence time, lipophilicity, and}

\section{lipid conjugation}

The pulmonary residence time of an IC is defined as the average time necessary for the absorption of a molecule of the drug into the systemic circulation. Lipophilicity and lipid conjugation are two different parameters that have effect on the absorption rate of an IC through pulmonary membranes and pulmonary residence time. The lipophilicity enables the transition of the IC through the phospholipid bilayer of the cell membranes and shows a positive correlation with pulmonary retention. Lipid conjugation or esterification of fatty acid takes 
place with the establishment of a reversible chemical bond between the IC and fatty acids in pulmonary cells and prolongs the pulmonary residence time providing a slow-release IC reservoir in the target tissue (Derendorf et al., 2006; Baptist and Reddy, 2009).

\subsection{Type of Inhaler Device}

Inhaler devices and their contents have an impact on the extent of IC exposure. Ideally, an inhaler device is expected to transfer a predetermined drug dose to the lungs with minimal oropharyngeal drug deposition and maximum drug proportion reaching the lung reducing the potential for oropharyngeal adverse effects, be easy to use especially in the elderly or physically/cognitively disabled patients, portable, reproducible, and cost effective (Roland et al., 2004; Irwin and Richardson, 2006; Rachelefsky et al., 2007; Hossny et al., 2016). Pressurised metered-dose inhalers (pMDIs) with a spacer device, metered dose inhalers (MDIs) with/without a spacer device, dry powder inhalers (DPIs), and nebulizers in jet/ultrasonic types are among the devices currently available for the delivery of ICs (Roland et al., 2004; Baptist and Reddy, 2009; Hossny et al., 2016). No ideal device is available for the administration of inhaled drugs at the present time.

Depending on the application technique of the patient, the amount of drug delivered varies between 7 and 20\% for pMDIs and $80 \%$ of the dose remains in the oropharynx (Newman et al., 1991; Lipworth, 1995). On the other hand, for DPIs $\leq 60 \%$ of the administered dose is deposited in the oropharynx (Selroos et al., 1994; Lipworth, 1995). The distance that the dispersed drug has to pass before being inhaled is increased by spacer (extension) devices. The use of these devices allows the aerosol to be slowed down, the size of the aerosol droplets to be reduced, the large non-respirable particles to be trapped, and thus the oropharyngeal effect of the drug is reduced.

There are studies on the effect of inhaler device type and local adverse effects of ICs. A meta-analysis revealed a 5 and 3 times greater risk for oral candidiasis compared to the placebo group when steroids were inhaled using MDI and DPI devices, respectively (Rachelefsky et al., 2007). In addition, nebulizers were asserted to be not less effective than MDIs with large-volume spacers for IC delivery in a systematic review (Cates et al., 2006).

2.4. Patient's Compliance with the Instructions for Administration

Incorrect inhalation technique is an important factor that causes inadequate delivery of the drug to the lungs, and oropharyngeal complications may limit the effectiveness of ICs due to the large amount of aerosol accumulated in the mouth (Kwah and Peters, 2019).

For the old chlorofluorocarbon (CFC)-driven pMDI devices which have the active drug included as a micronized suspension, the lack of shaking the device prior to use or between consecutive doses might cause the suspension to disperse inconveniently in the propellant and result in up to $50 \%$ reduction in the delivery of $\beta 2$-agonists and corticosteroids. On the other hand, shaking is not necessary for the modern hydrofluoroalkane (HFA)-driven pMDIs since the active drug is in true solution. There is no need to shake or invert the DPI devices following priming, and patients should be informed by prescribers to be aware of this (Buttini et al., 2014; Levy et al., 2016).

Holding the breath following inhaling from a pMDI or DPI has been found to be beneficial as it may enhance the inhaled drug deposition in the lungs. Therefore, following inhalation holding their breath for a minimum of 5 seconds is recommended to the patients (Thorsson, 1998; Levy et al., 2016).

Patients should avoid exhaling or blowing into DPI devices, as it will cause condensation and humidity and a decrease in the amount of fine-particles of the inhaled drug. Since humidity decreases their dispersal properties, DPI devices should be stored in a moisturefree environment (Thorsson, 1998; Holmes et al., 2015; Levy et al., 2016).

Amongst the inhaler devices, nebulizers require minimal patient technique and active cooperation, so their use may be preferred in children. MDIs combined with holding chambers and masks, as well as DPIs, are suitable for use in older children and adolescents that are cooperative (Cates et al., 2006; Baptist and Reddy, 2009; Hossny et al., 2016).

\section{Oral Candidiasis Related to Inhaled Corticosteroids}

The most commonly encountered oral fungal infection in humans is candidiasis and is manifested in various variants clinically varying from pseudomembranous, erythematous and hyperplastic, to median rhomboid glossitis, angular cheilitis and Candida-associated denture stomatitis (Ellepola et al., 2001; Fukushima et al., 2001; Komiyama et al., 2004; Gumru Tarcin, 2011; Patil et al., 2016).

The presence of a large number of predisposing factors that facilitate the transformation of commensal Candida into parasitic status is regarded as the main reason for the high oral candidiasis incidence. Among the reasons for the development of oral candidiasis as a relatively common disease are the emergence of Human Immunodeficiency Virus (HIV) infection, the increase in the prevalence of compromised patient groups in the public, common endocrine disorders such as diabetes mellitus, and the presence of nutritional deficiencies. Besides these factors, oral candidiasis may develop as an adverse effect following the use of agents such as broadspectrum antibiotics, cytotoxics, and corticosteroids (Toogood et al., 1980; Hanania et al., 1996; Kennedy et al., 2000; Ellepola et al., 2001; Roland et al., 2004; Gumru Tarcin, 2011; van Boven et al., 2013; Hossny et al., 2016; Patil et al., 2016; Erdoğan et al., 2019).

It has been reported that the incidence of Candida albicans (C. albicans) in the oral cavity $45-65 \%$ in 
newborns and healthy children, and $30-45 \%$ in healthy adults. Higher incidence rates have been reported for patients wearing removable dentures (50-65\%), residing in acute and long-term care facilities (65-88\%), with acute leukemia undergoing chemotherapy (90\%), and with HIV infection (95\%) (Hanania et al., 1995; Samaranayake and Samaranayake, 2001; Komiyama et al., 2004; Patil et al., 2016).

Clinically significant incidence rate of oral candidiasis is probably lower in adults (4-13\%) and in children (1-3\%) (Maxwell, 1990; Ming et al., 2019). With regular use of ICs in asthmatic patients, the rate of oropharyngeal candidiasis development as a potential adverse effect varies from 0 to $77 \%$, and the reason for this wide range is probably the differences in detection methodologies (Toogood et al., 1980; Spector et al., 1982; Maxwell, 1990; Toogood, 1990; Kennedy et al., 2000; Fukushima et al., 2001; Komiyama et al., 2004; Roland et al., 2004; Buhl, 2006; Irwin and Richardson, 2006; Thomas et al., 2010; van Boven et al., 2013; Dekhuijzen et al., 2016; Hejazi et al., 2016; Cheng et al., 2017; Sharma et al., 2018; Erdoğan et al., 2019; Ming et al., 2019).

\subsection{Mechanisms of Pathogenicity}

The mechanism by which C. albicans lead to development of oral candidasis includes the ability of mucous membrane adherence, pseudohyphae formation, and hydrolytic enzyme secretion. On the other hand, the defence of the oral cavity is based on non-specific immune mechanisms such as mucosal integrity, leukocytes, macrophages, and salivary components, and on specific immune mechanisms such as antibodies and cell-mediated immunity (Samaranayake and Samaranayake, 2001; Komiyama et al., 2004).

The mechanisms by which ICs cause oral candidiasis have not been clearly established. Because only 10 to $20 \%$ of the dose taken with the use of an inhaler reaches the lungs, while the rest remains in the oropharynx, the topical effects of ICs on the oral mucosa can be held responsible for this adverse effect (Maxwell, 1990; Newman et al., 1991; Lipworth, 1995; Pedersen and O’Byrne, 1997; Komiyama et al., 2004; Roland et al., 2004; Derendorf et al., 2006; Baptist and Reddy, 2009; Thomas et al., 2010; Hejazi et al., 2016; Erdoğan et al., 2019). Most of the inhaled drug remains in the oral cavity and oropharynx, and as a result it may affect the physiology of oral tissues (Godara et al., 2011).

Immunosuppressive and anti-inflammatory effects of steroids are considered to have a significant effect on the pathogenesis of oral candidiasis (Ellepola and Samaranayake, 2001; Thomas et al., 2010). This local adverse effect may be due to a decrease in local immune response which involves the inhibition of defence functions of neutrophils, macrophages, and Tlymphocytes at the surface of oral mucosa by IC particle deposition in the upper airways or related to a salivary glucose level increase that promote the candidal growth, proliferation, and adhesion to oral mucosal cells following IC accumulation in the oropharyngeal cavity
(Toogood, 1990; Hanania et al., 1995; Ellepola et al., 2001; Fukushima et al., 2003; Roland et al., 2004; Buhl, 2006; Rachelefsky et al., 2007; van Boven et al., 2013; Dekhuijzen et al., 2016; Cheng et al., 2017; Erdoğan et al., 2019; Ming et al., 2019). Several studies have shown that salivary IgA, histatin, defensin, and lactoferrin, which are contributing factors in local immunity, are associated with the formation of oral candidiasis (Helmerhorst et al., 2001; Samaranayake and Samaranayake, 2001; Sawaki et al., 2001). ICs may decrease total salivary IgA among these important host factors and patients with asthma having lower total salivary IgA levels show a tendency of developing oral candidiasis (Fukushima et al., 2003; Sharma et al., 2018).

Most DPIs contain 10-25 mg lactose monohydrate per dose as the carrier vehicle (Thomas et al., 2010). ICs have weak organic acidic structure and cannot be mainly metabolized by oral bacteria. This high lactose concentration results in increased salivary glucose level, enables higher steroid uptake in oral and laryngeal regions, and also promotes candidal growth, proliferation, and adhesion (Ellepola and Samaranayake, 2001; Thomas et al., 2010; Patil et al., 2016; Ullah et al., 2016; Sharma et al., 2018). Moreover, low pH levels due to high glucose concentration in saliva create a favourable environment for the secretion of potent extracellular enzymes such as aspartyl proteinases and phospholipases that contribute to the pathogenicity of Candida (Kargul et al., 1998; Wu and Samaranayake, 1999; Lenander-Lumikari et al., 2000; Ellepola and Samaranayake, 2001; Godara et al., 2011).

\subsection{Clinical Presentation}

Since Dennis and Itkin, who reported oral candidiasis in $20 \%$ of the asthmatic patients under treatment with inhalers containing dexamethasone, noted the relationship between ICs and oral candidiasis for the first time, a considerable amount of studies has emerged either confirming or disproving their observation (Dennis and Itkin, 1964). Because of the differences in study populations, absence of control groups, simultaneous use of antibiotics, previous treatment with steroids, and differences in sampling techniques, it is challenging to compare the studies in the literature (Ellepola and Samaranayake, 2001). However, it is widely agreed that oral candidal carriage and/or clinical infection as the pseudomembranous or erythematous variant is generally promoted by the use of ICs (Ellepola and Samaranayake, 2001). Patients who are carriers of Candida prior to IC use are more likely to develop oral candidiasis clinically (Spector et al., 1982).

Pseudomembranous candidiasis clinically appears as whitish creamy plaques looking like milk curds that can be removed from the surface by gentle wiping leaving painful erythematous or bleeding mucosal surfaces (Godara et al., 2011; Gumru Tarcin, 2011; Patil et al., 2016) (Figure 1). Patients often complain about regional discomfort such as alteration in taste sensation, tenderness, burning, and dysphagia since the 
pseudomembrane gets disrupted. Localized and painful erythematous areas are characteristic clinical findings of erythematous candidiasis (Kennedy et al., 2000; Godara et al., 2011; Gumru Tarcin, 2011; van Boven et al., 2013; Hossny et al., 2016). These two distinct clinical forms of oral candidiasis can be seen simultaneously (Figure 2).

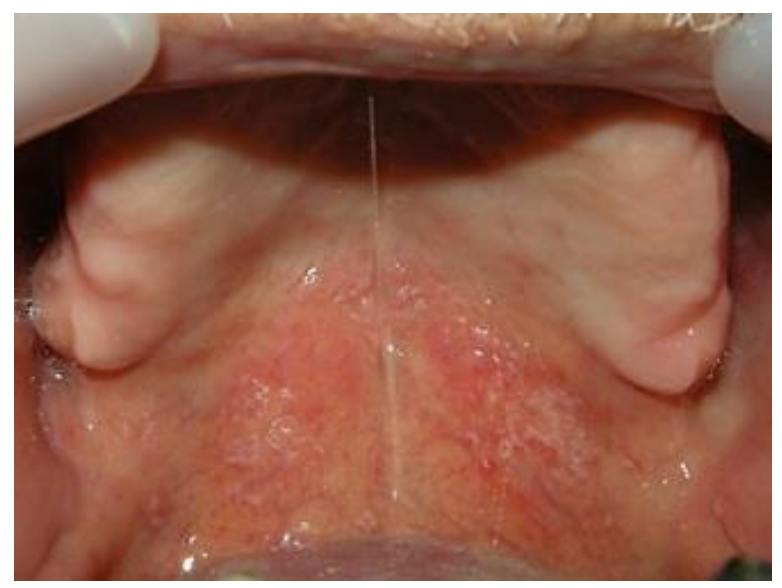

Figure 1. Pseudomembranous candidiasis in a 73-yearold patient on IC therapy for asthma and insulin therapy for diabetes mellitus.

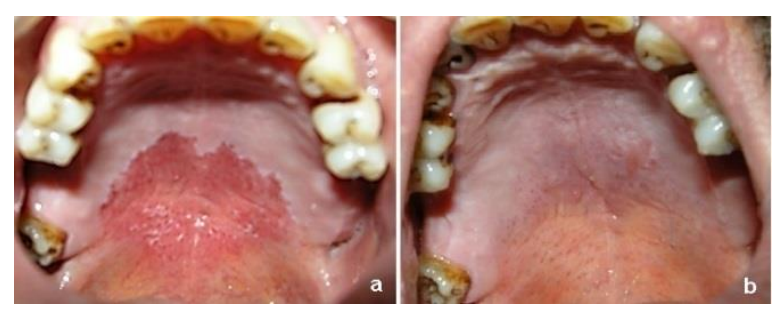

Figure 2. Concurrence of pseudomembranous and erythematous candidiasis in a 42-year-old asthmatic patient using IC (a).Recovery after the use of Nystatin oral suspension 4 times daily for a period of two weeks (b).

In all cases, candidiasis, the severity of which is commonly associated to daily dose and frequency of intake, is particularly limited to the oral mucosa exposed directly to the IC (Dennis and Itkin, 1964; Toogood et al., 1980; Ellepola and Samaranayake, 2001; Roland et al., 2004; Godara et al., 2011; Hossny et al., 2016) (Figure 3). The common sites are buccal mucosa, soft palate, oropharynx, and lateral aspects of tongue (Godara et al., 2011; Hossny et al., 2016; Patil et al., 2016).

\subsection{Treatment}

In general, the majorities of oral candidal infections are superficial and can be treated simply by controlled administration of topical antifungal agents, including the polyene drugs such as Nystatin and Amphotericin or azole group drugs such as Miconazole, Clotrimazole, Fluconazole, and Ketoconazole (Patil et al., 2016). Miconazole oral gels or mouthwashes containing Nystatin are considered as the main treatments for oral candidiasis (van Boven et al., 2013). Topical treatment with Nystatin oral suspension is recommended due to its effectiveness on inhibition of candidal growth in oral cavity (Epstein et al., 1986; Prentice, 1989; Hanania et al., 1995; Ellepola and Samaranayake, 2001; Fukushima et al., 2001; Thomas et al., 2010). Nystatin lozenges have been found to be as clinically and microbiologically effective as Nystatin suspensions (Thompson et al., 1986; Ellepola and Samaranayake, 2001). Antifungal vaginal suppositories, which are free of sugar, are recommended to be dissolved in the mouth in order to avoid dental caries (Regezi et al., 2016).

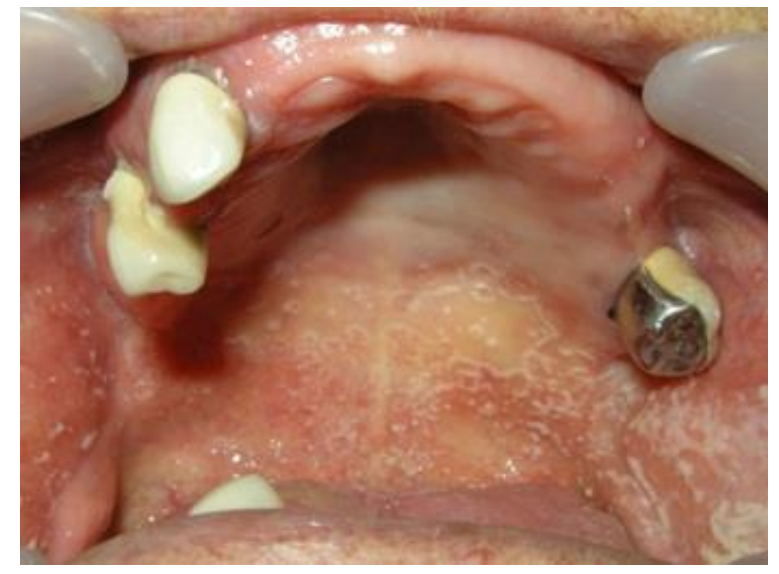

Figure 3. Pseudomembranous candidiasis in a 42-yearold patient on therapy for severe asthma, hypertension, and hypothyroidism.

However, a few oral candidiasis cases that have not responded to Nystatin have been reported in the literature in case of combination with triamcinolone acetonide (Martin et al., 1982; Ellepola and Samaranayake, 2001). The high in vitro resistance of the C. albicans isolates from these patients to combination of Nystatin and triamcinolone acetonide has been shown as the reason of this failure (Barkvoll and Attramadal, 1989). This finding is surprising because development of resistance to azole group is quite common in Candida, whereas Nystatin resistance is extremely rare (Ellepola and Samaranayake, 2001). The reason of Nystatin resistance can be explained by the inactivation of the antifungal agent when combined with triamcinolone acetonide. This mechanism is similar to how Nystatin is deactivated when combined with chlorhexidine (Barkvoll and Attramadal, 1989).

Local administration of Amphotericin $B$ and Methylrosaniline is recommended in patients who do not respond to Nystatin or Miconazole (van Boven et al., 2013). In addition, systemic antifungal administration is regarded as an alternative approach in patients not responding to local antifungal therapy. Oral Ketoconazole and intravenous Amphotericin B have been shown to be effective in such cases (Prentice, 1989; Ellepola and Samaranayake, 2001).

Echinocandins, such as Caspofungin, Micafungin, and Anidulafungin, are a newer class of antifungal agents available in intravenous formulations (Lombardi and 
Ouanounou, 2020). Due to some limitations of polyene, azole, and echinocandin antifungal agents such as toxicity, low selectivity, and emerging resistance, two new glucan synthesis inhibitors, Ibrexafungerp and Rezafungin, have been postulated as new antifungal agents effective especially in multidrug resistance (Lima et al., 2019; Lombardi and Ouanounou, 2020; Nivoix et al., 2020). In addition, among the novel antifungal treatment alternatives, such as probiotics, photodynamic therapy, and plant derivatives, the use of probiotics seems to be the most remarkable. Probiotics, used alone or in combination with other antifungal agents such as Nystatin, have been reported to have the potential to effectively reduce Candida levels (Li et al., 2014; Ishikawa et al., 2015; Matsubara et al., 2016; Lombardi and Ouanounou, 2020).

Oral candidiasis is usually not severe and responds well to appropriate and effective treatment with good prognosis, but may sometimes be bothersome for the patients (Spector et al., 1982; Ellepola and Samaranayake, 2001; Patil et al., 2016).

\subsection{Preventive Strategies}

A series of precautions can be taken to minimize the risk of developing oral candidiasis in patients undergoing inhalation therapy (Toogood et al., 1980; Epstein et al., 1986; Prentice, 1989; Maxwell, 1990; Toogood, 1990; Selroos et al., 1994; Hanania et al., 1995; Lipworth, 1995; Kargul et al., 1998; Ellepola and Samaranayake, 2001; Roland et al., 2004; Yokoyama et al., 2005; Irwin and Richardson, 2006; Yokoyama et al., 2006; Rachelefsky et al., 2007; Thomas et al., 2010; Godara et al., 2011; Hejazi et al., 2016; Patil et al., 2016; Cheng et al., 2017; Ming et al., 2019):

- Education of the patients about the potential adverse effects of inhalation therapy.

- Emphasizing the use of a spacer device (holding chamber or extension device) to minimize the accumulation of the ICs in the oral cavity and oropharynx. Using a spacer device combined with the inhaler can diminish the development of local adverse effects of ICs, such as oral candidiasis, as it reduces the oropharyngeal accumulation and increases the access to the lungs.

- Making regular dental check-up appointments at least every 6 months.

- Recommending the patients, who use high doses of ICs with DPIs, to rinse their mouths using water, neutral $\mathrm{pH}$ or basic mouth rinses (milk, water, sodium bicarbonate, neutral sodium fluoride $0.05 \%$ mouth rinses, liquid antacids), or antimicrobial mouth rinses immediately following each use of ICs especially before bedtime. This is to neutralize the acidic pH of the DPIs and reduce candidal colonization.

- Increasing the salivary flow rate in patients with low salivary output may also ensure the reduction oral Candida. In this regard, the use of sialagogue drugs or sugar-free chewing gums can be recommended.

- Controlled topical antimycotic use, such as Nystatin, has also been shown to prevent oral candidiasis due to prolonged steroid therapy damaging mucosal barriers. However, recurrences may occur if the antifungal is discontinued. Therefore, it is necessary to continue using Nystatin over the course of a steroid treatment regimen.

\section{Conclusion}

Local adverse effects of ICs are often negligible and their effect on the patient's overall health is minimal. However, adverse effects of ICs cause fear in patients and local adverse effects may negatively affect the compliance with the therapy. The fact that some of the local adverse effects depend on the dose and some on the device reveals the necessity to choose the lowest effective dose and the optimum device of an IC. The relationship between asthma and oral health should be considered by the dental practitioners and measures should be taken to avoid adverse effects on oral tissues.

\section{Author Contributions}

BG; contributed to study conception and design. BG and MPA; drafted, reviewed, and approved the final version of the manuscript.

\section{Conflict of Interest}

The authors declare that there is no conflict of interest.

\section{References}

Baptist AP, Reddy RC. 2009. Inhaled corticosteroids for asthma: are they all the same? J Clin Pharm Ther, 34(1): 1-12.

Barkvoll P, Attramadal A. 1989. Effect of nystatin and chlorhexidine digluconate on Candida albicans. Oral Surg Oral Med Oral Pathol, 67(3): 279-281.

Barnes PJ, Adcock IM. 2003. How do corticosteroids work in asthma? Ann Intern Med, 139(5 Pt 1): 359-370.

Buhl R. 2006. Local oropharyngeal side effects of inhaled corticosteroids in patients with asthma. Allergy, 61(5): 518526.

Buttini F, Miozzi M, Balducci AG, Royall PG, Brambilla G, Colombo P, Bettini R, Forbes B. 2014. Differences in physical chemistry and dissolution rate of solid particle aerosols from solution pressurised inhalers. Int J Pharm, 465(1-2): 42-51.

Cates CJ, Bestall J, Adams N. 2006. Holding chambers versus nebulisers for inhaled steroids in chronic asthma. Cochrane Database Syst Rev, 1: CD001491.

Cheng T, Yong L, Zhang H, Chen L, Tu J, Hui X, Cheng Q, Wan H. 2017. Incidence of oral candidiasis is associated with inhaled corticosteroids in Chinese patients: a systematic review and meta-analysis. Int J Clin Exp Med, 10(3): 5546-5560.

Dahl R. 2006. Systemic side effects of inhaled corticosteroids in patients with asthma. Respir Med, 100(8): 1307-1317.

Dekhuijzen PNR, Batsiou M, Bjermer L, Bosnic-Anticevich S, Chrystyn H, Papi A, Rodríguez-Roisin R, Fletcher M, Wood L, Cifra A, Soriano JB, Price DB. 2016. Incidence of oral thrush in patients with COPD prescribed inhaled corticosteroids: effect of drug, dose, and device. Respir Med, 120: 54-63.

Dennis M, Itkin IH. 1964. Effectiveness and complications of aerosol dexamethasone phosphate in severe asthma. J Allergy, 35: 70-76.

Derendorf H, Nave R, Drollmann A, Cerasoli F, Wurst W. 2006. Relevance of pharmacokinetics and pharmacodynamics of 


\section{Black Sea Journal of Health Science}

inhaled corticosteroids to asthma. Eur Respir J, 28(5): 10421050.

Derendorf H. 1997. Pharmacokinetic and pharmacodynamic properties of inhaled corticosteroids in relation to efficacy and safety. Respir Med, 91(Suppl A): 22-28.

Ellepola AN, Samaranayake LP. 2001. Inhalational and topical steroids, and oral candidosis: a mini review. Oral Dis, 7(4): 211-216.

Epstein JB, Komiyama K, Duncan D. 1986. Oral topical steroids and secondary oral candidiasis. J Oral Med, 41(4): 223-227, 273.

Erdoğan T, Karakaya G, Kalyoncu AF. 2019. The frequency and risk factors for oropharyngeal candidiasis in adult asthma patients using inhaled corticosteroids. Turk Thorac J, 20(2): 136-139.

Fukushima C, Matsuse H, Tomari S, Obase Y, Miyazaki Y, Shimoda T, Kohno S. 2003. Oral candidiasis associated with inhaled corticosteroid use: comparison of fluticasone and beclomethasone. Ann Allergy Asthma Immunol, 90(6): 646651.

Fukushima C, Shimoda T, Kawano T, Tomari S, Mitsuta K, Obase Y, Matsuo N, Matsuse H, Kohno S. 2001. Effects of amphotericin B gargles on oral colonization of Candida albicans in asthmatic patients on steroid inhalation therapy. Respiration, 68(5): 465-470.

Godara N, Godara R, Khullar M. 2011. Impact of inhalation therapy on oral health. Lung India, 28(4): 272-275.

Gumru Tarcin B. 2011. Oral candidosis: aetiology, clinical manifestations, diagnosis and management. MÜSBED, 1(2): 140-148.

Hanania NA, Chapman KR, Kesten S. 1995. Adverse effects of inhaled corticosteroids. Am J Med, 98(5 Pt 1): 196-208.

Hejazi ME, Shafiifar A, Mashayekhi S, Sattari M. 2016. Evaluation of proper usage of glucocorticosteroid inhalers and their adverse effects in asthmatic patients. Tanaffos, 15(1): 9-16.

Helmerhorst EJ, Troxler RF, Oppenheim FG. 2001. The human salivary peptide histatin 5 exerts its antifungal activity through the formation of reactive oxygen species. Proc Natl Acad Sci U S A, 98(25): 14637-14642.

Holmes MS, Seheult JN, O'Connell P, D'Arcy S, Ehrhardt C, Healy AM, Costello RW, Reilly RB. 2015. An acoustic-based method to detect and quantify the effect of exhalation into a dry powder inhaler. J Aerosol Med Pulm Drug Deliv, 28(4): $247-$ 253

Hossny E, Rosario N, Lee BW, Singh M, El-Ghoneimy D, Soh JY, Le Souef P. 2016. The use of inhaled corticosteroids in pediatric asthma: update. World Allergy Organ J, 9: 26.

Irwin RS, Richardson ND. 2006. Side effects with inhaled corticosteroids: the physician's perception. Chest, $130(1$ Suppl): 41S-53S.

Ishikawa KH, Mayer MP, Miyazima TY, Matsubara VH, Silva EG, Paula CR, Campos TT, Nakamae AE. 2015. A multispecies probiotic reduces oral Candida colonization in denture wearers. J Prosthodont, 24(3): 194-199.

Kargul B, Tanboga I, Ergeneli S, Karakoc F, Dagli E. 1998. Inhaler medicament effects on saliva and plaque $\mathrm{pH}$ in asthmatic children. J Clin Pediatr Dent, 22(2): 137-140.

Kennedy WA, Laurier C, Gautrin D, Ghezzo H, Paré M, Malo JL, Contandriopoulos AP. 2000, Occurrence and risk factors of oral candidiasis treated with oral antifungals in seniors using inhaled steroids. J Clin Epidemiol, 53(7): 696-701.

Komiyama EY, Ribeiro PM, Junqueira JC, Koga-Ito CY, Jorge AO. 2004. Prevalence of yeasts in the oral cavity of children treated with inhaled corticosteroids. Braz Oral Res, 18(3):
197-201.

Kwah JH, Peters AT. 2019. Asthma in adults: Principles of treatment. Allergy Asthma Proc, 40(6): 396-402.

Lenander-Lumikari M, Soderling E, Loimaranta V, Ampula L. 2000. Effect of inhaled corticosteroids on plaque $\mathrm{pH}$. Caries Res, 34: 348.

Levy ML, Dekhuijzen PN, Barnes PJ, Broeders M, Corrigan CJ, Chawes BL, Corbetta L, Dubus JC, Hausen T, Lavorini F, Roche N, Sanchis J, Usmani OS, Viejo J, Vincken W, Voshaar T, Crompton GK, Pedersen S. 2016. Inhaler technique: facts and fantasies. A view from the Aerosol Drug Management Improvement Team (ADMIT). NPJ Prim Care Respir Med, 26: 16017.

Li D, Li Q, Liu C, Lin M, Li X, Xiao X, Zhu Z, Gong Q, Zhou H. 2014. Efficacy and safety of probiotics in the treatment of Candidaassociated stomatitis. Mycoses, 57(3): 141-146.

Lima SL, Colombo AL, de Almeida Junior JN. 2019. Fungal cell wall: Emerging antifungals and drug resistance. Front Microbiol, 10: 2573.

Lipworth BJ. 1995. New perspectives on inhaled drug delivery and systemic bioactivity. Thorax, 50(2): 105-110.

Lombardi A, Ouanounou A. 2020. Fungal infections in dentistry: Clinical presentations, diagnosis, and treatment alternatives. Oral Surg Oral Med Oral Pathol Oral Radiol, 130(5): 533-546.

Martin MV, Dinsdale RC. 1982. Nystatin-resistance of candida albicans isolates from two cases of oral candidiasis. Br J Oral Surg, 20(4): 294-298.

Matsubara VH, Bandara HM, Mayer MP, Samaranayake LP. 2016. Probiotics as antifungals in mucosal candidiasis. Clin Infect Dis, 62(9): 1143-1153.

Maxwell DL. 1990. Adverse effects of inhaled corticosteroids. Biomed Pharmacother, 44(8): 421-427.

Ming SWY, Haughney J, Ryan D, Patel S, Ochel M, d'Alcontres MS, Thornhill S, Kocks JWH, Price D. 2019. Comparison of adverse events associated with different spacers used with non-extrafine beclometasone dipropionate for asthma. NPJ Prim Care Respir Med, 29(1): 3.

Newman SP, Weisz AW, Talaee N, Clarke SW. 1991. Improvement of drug delivery with a breath actuated pressurised aerosol for patients with poor inhaler technique. Thorax, 46(10): 712-716.

Nivoix Y, Ledoux MP, Herbrecht R. 2020. Antifungal therapy: New and evolving therapies. Semin Respir Crit Care Med 41(1): 158-174.

Patil A, Susmitha HR, Basappa S, Mahesh MS. 2016. Druginduced oral candidiasis: a case report. IJSS Case Rep Rev, 2(12): 1-4.

Pedersen S, O'Byrne P. 1997. A comparison of the efficacy and safety of inhaled corticosteroids in asthma. Allergy, 52(39 Suppl): 1-34.

Prentice AG. 1989. Oral and gastrointestinal candidosis: prophylaxis during immunosuppressive therapy. Mycoses, 32(Suppl 2): 42-46.

Rachelefsky GS, Liao Y, Faruqi R. 2007. Impact of inhaled corticosteroid-induced oropharyngeal adverse events: results from a meta-analysis. Ann Allergy Asthma Immunol, 98(3): 225-238.

Regezi JA, Sciubba JJ, Jordan RCK. 2016. Oral Pathology, Clinical Pathologic Correlations. Elsevier, St. Louis, Missoouri, USA 7th ed., pp 108.

Roland NJ, Bhalla RK, Earis J. 2004. The local side effects of inhaled corticosteroids: current understanding and review of the literature. Chest, 126(1): 213-219.

Samaranayake YH, Samaranayake LP, Pow EH, Beena VT, Yeung KW. 2001. Antifungal effects of lysozyme and lactoferrin 
against genetically similar, sequential Candida albicans isolates from a human immunodeficiency virus-infected southern Chinese cohort. J Clin Microbiol, 39(9): 3296-3302.

Samaranayake YH, Samaranayake LP. 2001. Experimental oral candidiasis in animal models. Clin Microbiol Rev, 14(2): 398429.

Sawaki K, Mizukawa N, Yamaai T, Fukunaga J, Sugahara T. 2002. Immunohistochemical study on expression of alpha-defensin and beta-defensin-2 in human buccal epithelia with candidiasis. Oral Dis, 8(1): 37-41.

Selroos O, Backman R, Forsén KO, Löfroos AB, Niemistö M, Pietinalho A, Aikäs C, Riska H. 1994. Local side-effects during 4-year treatment with inhaled corticosteroids - a comparison between pressurized metered-dose inhalers and Turbuhaler. Allergy, 49(10): 888-890.

Sharma S, Gaur P, Gupta S, Kant S. 2018. Impact of asthma on oral health: a review. Int J Recent Sci Res, 9(5): 26512-26514.

Spector SL, Wangaard C, Bardana EJ Jr. 1982. The use of cultures and immunologic procedures to predict oropharyngeal candidiasis in patients on steroid aerosols. Clin Allergy, 12(3): 269-278.

Thomas MS, Parolia A, Kundabala M, Vikram M. 2010. Asthma and oral health: a review. Aust Dent J, 55(2): 128-133.

Thompson PJ, Wingfield HJ, Cosgrove RF, Hughes BO, TurnerWarwick ME. 1986. Assessment of oral candidiasis in patients with respiratory disease and efficacy of a new nystatin formulation. Br Med J (Clin Res Ed), 292(6537): 1699-700.

Thorsson L, Kenyon C, Newman SP, Borgström, L. 1998. Lung deposition of budesonide in asthmatics: a comparison of different formulations. Int J Pharm, 168: 119-127.

Toogood JH, Jennings B, Greenway RW, Chuang L. 1980. Candidiasis and dysphonia complicating beclomethasone treatment of asthma. J Allergy Clin Immunol, 65(2): 145-153.

Toogood JH. 1990. Complications of topical steroid therapy for asthma. Am Rev Respir Dis, 141(2 Pt 2): S89-S96.

Ullah Z, Iqbal Z, Anwar K. 2016. Frequency of oral candidiasis in ashtma patients using inhaled corticosteroids by different inhaler devices. Pak J Chest Med, 22(1): 8-11.

van Boven JF, de Jong-van den Berg LT, Vegter S. 2013. Inhaled corticosteroids and the occurrence of oral candidiasis: a prescription sequence symmetry analysis. Drug Saf, 36(4): 231-236.

Wu T, Samaranayake LP. 1999. The expression of secreted aspartyl proteinases of Candida species in human whole saliva. J Med Microbiol, 48(8): 711-720.

Yokoyama H, Nakajima Y, Yamamura Y, Iga T, Yamada Y. 2005. Investigation of mouth washing by patients after inhaling corticosteroids. Yakugaku Zasshi, 125(5): 455-461.

Yokoyama H, Yamamura Y, Ozeki T, Iga T, Yamada Y. 2006. Influence of mouth washing procedures on the removal of drug residues following inhalation of corticosteroids. Biol Pharm Bull, 29(9): 1923-1925.

Yudt MR, Cidlowski JA. 2002. The glucocorticoid receptor: coding a diversity of proteins and responses through a single gene. Mol Endocrinol, 16(8): 1719-1726. 